\title{
DISEÑO DE SITUACIONES DE MODELACIÓN. UNA PROPUESTA PARA LA FORMACIÓN INICIAL DE DOCENTE DE MATEMÁTICA
}

\section{DESIGN OF MODELING SITUATIONS. APROPOSAL FOR THE INITIAL TRAINING OF MATHEMATICS TEACHERS}

\section{DANIELA SOTO*}

\author{
Rec.: 05-11-2019 Acept.: 22-06-2020 Publ.: 30-jun-2020
} DOI: http://doi.org/10.29035/ucmaule.58.107

\section{RESUMEN}

Se presenta una propuesta para el diseño de situaciones de modelación, aplicado en una asignatura de didáctica impartida en un programa de formación inicial del docente de matemática (FIDM). La propuesta contempla tres fases; problematización de la modelación, reconocimiento de una comunidad, y diseño, aplicación y análisis de una situación de modelación. Se documenta la problemática del diseño de situaciones de modelación, investigaciones sobre la modelación en la Matemática Educativa y la modelación desde la teoría que fundamentan la propuesta: la Teoría Socioepistemológica. Como resultado se reporta un diseño propuesto por futuras profesoras de matemáticas.

Palabras clave: Situaciones de modelación, Socioepistemológica, Formación inicial de profesores de matemática.

\begin{abstract}
A proposal for the design of modeling situations is presented, there applied in a didactic subject taught in an initial training program for the mathematics teacher (FIDM). The proposal includes three phases; problematization of modeling, recognition of a community, and design, application and analysis of a modeling situation. It is reported the problematic of the design of modeling situations, research on modeling in Educational Mathematics and modeling from the theory underlying the proposal: Socioepistemological Theory. As a result, a design proposed by future mathematics teachers is reported.
\end{abstract}

Key words: Modelling situations, Socioepistemological, Initial training of math teachers

* Universidad de Santiago de Chile. daniela.soto.s@usach.cl 


\section{Antecedentes}

Se presenta una propuesta para el diseño y análisis de situaciones de modelación en la formación inicial del docente de matemáticas (FIDM), desde la Teoría Socioepistemológica (TSE) (Cantoral, 2013, Cordero, 2016). Los fundamentos de esta propuesta radican en la problematización del saber matemático, la comunidad de conocimiento, el uso de la gráfica y el diseño de situaciones de modelación a partir del mantenimiento de rutinas y crisis.

La FIDM es una línea de investigación robusta dentro de la Matemática Educativa (ME) o Didáctica de la Matemática, esto queda reflejado en la gran cantidad de investigaciones desarrolladas, que presentan modelos alternativos de formación para promover mejoras en la FIDM. Alsina (2010) señala que entre estos modelos se encuentran: aprendizaje cooperativo, el colaborativo, el basado en la resolución de problemas, el basado en proyectos, el heurístico, el holístico o el aprendizaje reflexivo, entre otros.

Alsina (2010) profundiza sobre el aprendizaje reflexivo, entendiéndolo como aquel que se desarrolla con la práctica y que permite la interacción, reflexión y contraste de concepciones, creencias y de la teoría- práctica, para que el futuro profesor de matemáticas (FPM) pueda coconstruir y reconstruir sus conocimiento sobre la enseñanza y aprendizaje.

Llinares (2007) propone un modelo que promueve la utilización de variados entornos de aprendizaje para la formación del profesor de matemáticas. Estos entornos son oportunidades para que el FPM genere nuevo conocimiento y destrezas, de tal forma que potencie la capacidad para seguir aprendiendo desde la práctica. Llinares, Valls \& Roig (2008) presentan como ejemplo el ámbito específico del diseño de entornos virtuales de aprendizaje interactivos con videos.

Específicamente, sobre la FIDM y la modelación matemática se han documentado varias experiencias de cursos y/o asignaturas en la FIDM.

Villa-Ochoa (2016) promueve el análisis de modelos como una manera de introducir a los Futuros Profesores de Matemática (FPM) en la modelación matemática escolar. Señala que el análisis de modelos además de permitir al estudiante activar conocimientos y procedimientos matemáticos, le permite reflexionar sobre los usos sociales del modelo y reconocer maneras alternativas de producir conocimiento matemático en el aula. 
Huincahue, Borromeo-Ferri \& Mena-Lorca (2018) reportan los resultados de un curso sobre la modelación en la FIDM. El curso considera cuatro dimensiones: creencias y concepciones de la modelación matemática, elementos de la investigación en modelación matemática, resolución de tareas de modelación matemática y creación de tareas de modelación.

Méndez (2016) reporta una experiencia con un curso en la FIDM, la cual se caracteriza por el diseño propio y rediseño de actividades de modelación, fundamentados en la TSE y analizados a través de una ingeniería didáctica. El proceso descrito por esta autora contempla cuatro momentos, señalados en el siguiente esquema:

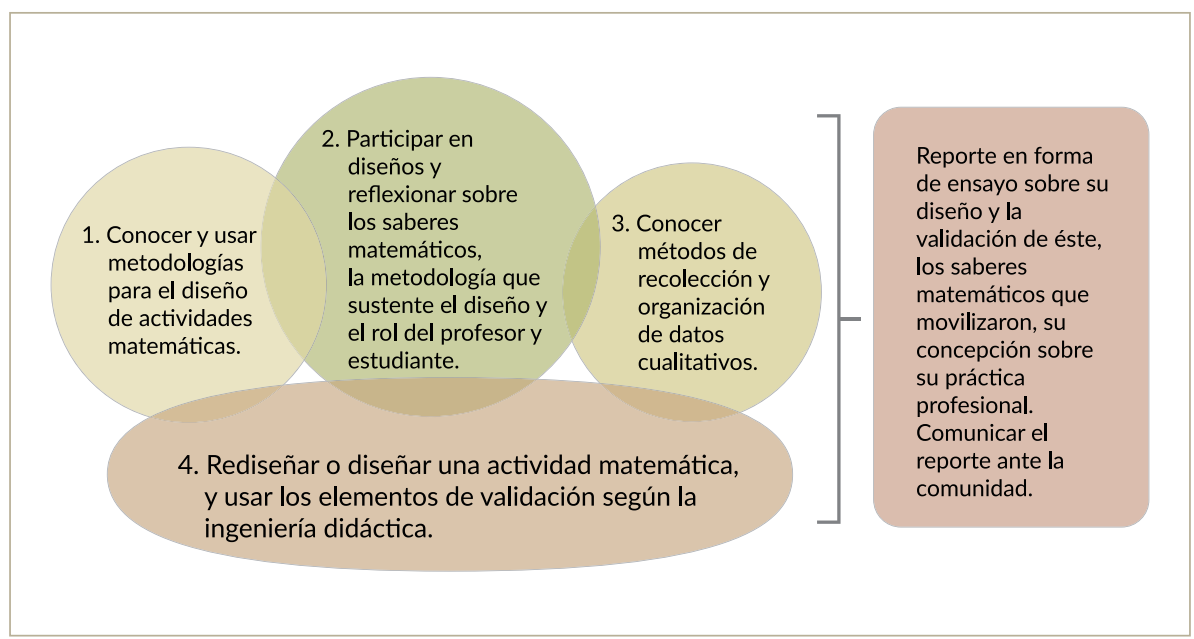

Ilustración 7. Esquema del proyecto de Méndez (2016).

Borromeo-Ferri (2014) presenta una propuesta para la enseñanza de la modelación en la formación de profesores de matemática, tanto en el nivel inicial como en servicio. Se contemplan cuatro dimensiones: teórica, de la tarea, de planificación y diagnóstica. Esta autora plantea que la dimensión teórica promueve la discusión sobre lo que se concibe por modelación matemática desde diferentes perspectivas, además de reflexionar sobre algunos ciclos de modelación. En la dimensión de la tarea, los profesores desarrollan análisis cognitivos de tareas de modelación, distinguiendo "buenas tareas". En la dimensión de planeación, se confronta la teoría con la práctica, se planifica la aplicación de la actividad de modelación. En la dimensión de diagnóstico, 
los profesores deben identificar errores o dificultes de los estudiantes con respecto al cumplimento del ciclo y de la actividad de modelación.

La propuesta que se presenta en este documento si bien considera en mayor o menor grado, los elementos expuestos anteriormente, como: la reflexión sobre las investigaciones de la ME, el contraste entre las creencias y concepciones de los FPM con las teorías, el diseño de situaciones o actividades de modelación, la aplicación del diseño de modelación y el análisis de modelos como una forma de introducir al FPM en la modelación. Se diferencia de ellas, al considerar a la "la gente" en la construcción del conocimiento matemático y por tanto, es fundamental que los y las FPM reconozcan las características de la comunidad de conocimiento para generar situaciones de modelación.

La propuesta se organiza en tres fases: problematización de la modelación, reconocimiento de una comunidad, y diseño, aplicación y análisis de una situación de modelación.

En las siguientes secciones se contempla profundizar acerca de la problemática de la modelación en la educación matemática, diferentes perspectivas sobre la modelación, la modelación desde la TSE, la presentación de la propuesta y, a modo de resultado, el reporte de un diseño elaborado por FPM.

\section{La problemática}

El currículo nacional chileno propone a la modelación como una de las cuatro habilidades para desarrollar el pensamiento matemático, junto a: representar, resolver problemas, argumentar y comunicar. Señala que la modelación permite "construir un modelo físico o abstracto que capture parte de las características de la realidad, para estudiarla, modificarla y/o evaluarla" (MINEDUC, 2015, p. 98).

Actividades, como la que se presentan en la ilustración 2, se pueden encontrar en documentos oficiales. Estas buscan constituir marcos de referencia para el profesor de matemática y el desarrollo de la modelación en el aula: 
2) Asocie cada función dada con su correspondiente esbozo de gráfica uniendo con una linea:
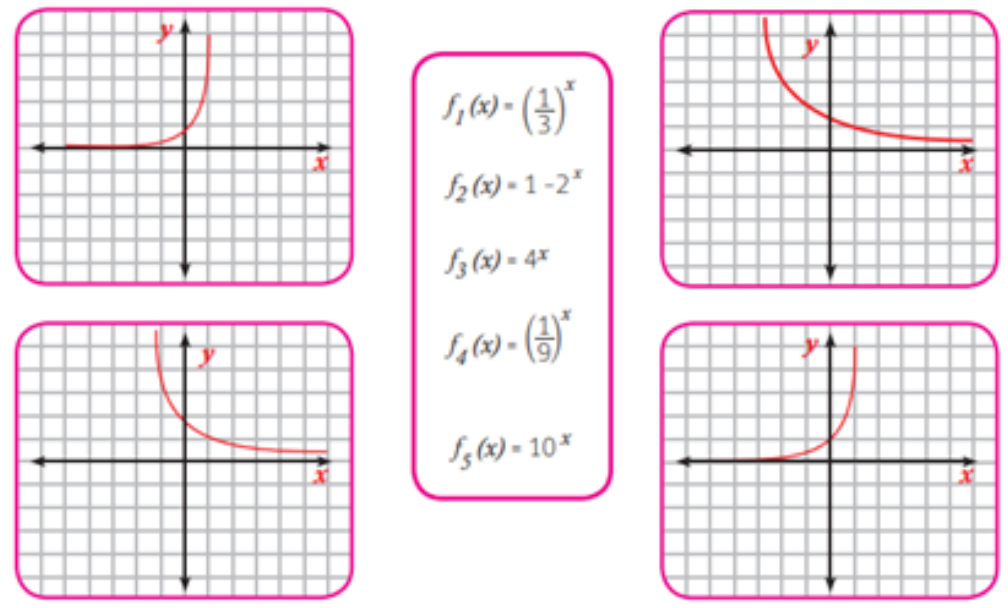

Ilustración 2. Huircán \& Carmona (2013, p. 14).

Esta actividad, extraída de Huircán \& Carmona (2013), si bien, permite la transformación entre representaciones de la función exponencial, carece de la conexión entre realidad y matemática, aspecto fundamental de la modelación (Blum \& Borromeo-Ferri, 2009, Cordero, 2016, Blomhøj, 2009, Kaiser, Sriraman, Blomhøj \& García, 2007).

Por otro lado, es común encontrar en los documentos oficiales propuestos por el Ministerio de Educación chileno, problemas que pretenden desarrollar la habilidad de modelar: 
2. Una empresa automotriz quiere proyectar la venta de dos modelos de autos para el resto del año, considerando que a fines de febrero se han vendido 90 unidades del modelo $A$ y 60 del modelo $B$. Para los próximos meses, se estima que la venta mensual del modelo $\mathrm{A}$ será de 15 autos y del modelo $\mathrm{B}$, de 20 autos. Se quiere saber el mes en el cual la venta del modelo B podría igualar la venta del modelo $\mathrm{A}$.

a. Completan la siguiente tabla:

\begin{tabular}{c|c|c|c|c|c|c|c|c|c|c}
\hline FEB & MAR & ABR & MAY & JUN & JUL & AGO & SEP & OCT & NOV & DIC \\
\hline 90 & & & & & & & & & & \\
\hline 60 & & & & & & & & & & \\
\hline
\end{tabular}

b. Confeccionan el gráfico eligiendo los ejes y la escala que muestra el desarrollo de la venta de ambos modelos de auto, y determinan el mes en el cual la venta del modelo $\mathrm{B}$ iguala la venta del modelo $\mathrm{A}$.

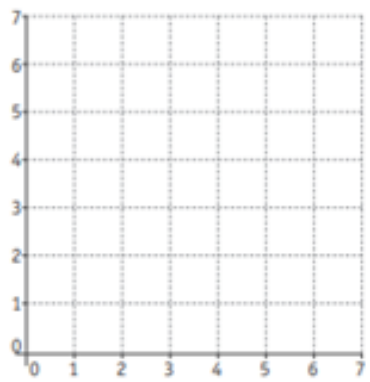

- Elaboran las ecuaciones de las funciones afines que modelan la venta de ambos tipos de auto. La variable independiente $x$ representa los meses $y$ la variable dependiente $y$ representa el número de autos vendidos.

Ilustración 3. Matemática, Programa de estudio, primero medio (MINEDUC, 2016, p. 105)

En el programa de estudio de primer año medio (14-15 años) este problema (Ilustración 3) se presenta como una actividad que permite el desarrollo de la habilidad de modelar. El problema se enmarca dentro de un contexto, la instrucción permite que los estudiantes transiten entre representaciones (tabular, gráfica y algebraica), sin embargo, las tareas no permiten que los estudiantes desarrollen conjeturas o transformaciones del fenómeno estudiado.

Las actividades señaladas expresan algunas de las tareas de modelación que se propician en la educación matemática actual y, por tanto, devela, en cierto grado, el desarrollo de la modelación en el aula.

\section{Modelar}

funcional para resc problemas y representar fenómenos cotidianos $y$ cientifices. (OA h) 
Ahora bien, investigaciones (Aravena, 2016, Huincahue \& Mena-Lorca, 2014, Vargas, Escalante \& Carmona, 2018) dan cuenta de las problemáticas asociadas a los procesos de modelación en el aula. Se documenta desde la escasa o nula presencia de este tipo de actividades en la clase de matemáticas, hasta las dificultades que presentan los FPM en la construcción de diseños de modelación.

Si nos adentramos en los programas de FIDM en Chile, se evidencia un escaso señalamiento hacia la modelación en las asignaturas que ofrecen (Vilches, Soto \& Silva-Crocci, 2019), en otras palabras se observa que no es claro el estatus de la modelación en la formación del FPM.

Soto, Silva-Crocci, Vergara \& Barve (2018) desarrollaron una investigación que evidenció que los profesores de matemáticas de una comunidad escolar, no consideran en sus planificaciones e instrumentos evaluativos la habilidad de modelar, es decir, en sus clases de matemática la modelación no está presente. Al ser cuestionados acerca de esto, los profesores señalan la falta de herramientas teóricas y prácticas para abordar esta habilidad. Esta experiencia permitió delinear estrategias en conjunto entre un programa de FIDM y la comunidad escolar de este estudio; una de esas estrategias es la propuesta que se presenta en este documento.

De esta forma, la necesidad por generar situaciones de modelación para el aula e introducir tempranamente al profesor en el diseño, provocó que se levantará una propuesta alternativa para que los FPM diseñen situaciones de modelación, considerando las características propias de una comunidad.

\section{La modelación}

Desde la ME existen variadas concepciones acerca de la Modelación en la educación matemática. Para profundizar se puede revisar Blomhøj (2009), quien presenta una sistematización de seis perspectivas de modelación en las investigaciones. Dentro de la literatura se destaca la perspectiva de modelación de Blum \& Borromeo-Ferri (2009). Esta concibe a la modelación como el proceso de traducción entre el mundo real y el mundo matemático. Se propone un ciclo de modelación, que permite establecer siete pasos cognitivos por los cuales debe transitar un sujeto al resolver una tarea de modelación. Si bien las investigaciones desarrolladas bajo este modelo han dejado claro que el camino no es necesariamente lineal. Se espera que el estudiante complete el ciclo, es decir logre transitar desde el mundo real al mundo matemático y luego vuelva al mundo real. 
Huincahue, Borromeo \& Mena-Lorca (2018) explican este proceso de la siguiente forma: la captura de la realidad a través de sus datos transforma la observación en una construcción matemática para analizar, e incluso, predecir la diversidad misma que la realidad permite; estableciendo un ciclo de validación en la matemática y en el mismo fenómeno.

Investigaciones en Chile como las de Aravena, Camaño \& Giménez (2008), Huincahue \& Mena-Lorca, (2014) y Solar, Azcárate \& Deulofeu (2012) señalan la necesidad de implementar la modelación en el aula. Aravena (2016), por ejemplo, señala que es fundamental la aplicación del conocimiento matemático a situaciones reales que estén en estrecha relación con la temática de estudio y sea susceptible a la formulación matemática propuesta. En particular, esta autora ha promovido el trabajo con estudiantes de la región del Maule (Chile), considerando la realidad cercana de los estudiantes para plantear resolución de problemas a través de proyectos.

Arrieta \& Díaz (2016) señalan que la modelación debe ser una práctica con vivencia, la cual puede ser una actividad de las comunidades y no necesariamente académica. Por ejemplo, la actividad del cardiólogo, cuando diagnostica y prescribe un tratamiento a un paciente con alteraciones en su corazón, lo realiza a partir de una gráfica llamada electrocardiograma, o de una tabla de datos obtenida desde un test de esfuerzo, lo cual es modelación.

Estos autores señalan que no se puede desvincular los modelos respecto de quien modela. En el caso del electrocardiograma, para un niño solo representa un papel con rayas, mientras que para el cardiólogo es un instrumento que le permite intervenir una realidad. La Modelación desde esta perspectiva es una práctica de articulación de dos entes: el modelo y lo modelado. A esto lo denominan Dipolo Modélico.

Villa-Ochoa (2016) señala que una forma de introducir a la FIDM, es el análisis de modelos. Para explicar esto, se hace referencia a una situación de análisis de un modelo de gestación de un bebé, en el que se utilizan diferentes representaciones, como: tabular, graficar y lenguaje natural. Se reconocen tres momentos en el análisis del modelo:

1. Acercamiento a las representaciones.

2. Profundización del fenómeno.

3. Reflexionar sobre los resultados. 
Para el análisis de modelos Villa-Ochoa (2016) plantean la aplicación de la tríada de Giere (1999): objeto que se modela, relación de representación y el objeto que modela, además del usuario. Cuando el usuario es el profesor en formación, éste adopta un estatus diferente. Puede constituirse en otro sujeto que debe comprender no solo los aspectos de las representaciones, lo modelado y el modelo, sino también todo lo que implica que la modelación sea una herramienta didáctica. En este sentido, el profesor es otro usuario, pero su relación con los objetos presentes en la tríada es diferente al usuario habitual.

En síntesis, a través de una breve revisión de referentes teóricos de la modelación, se pueden identificar algunos argumentos compartidos, como: la relación entre la matemática y la realidad, el reconocimiento del sujeto que modela (estudiante, profesor, FPM, entre otros) y la transformación de la realidad a partir del modelo o viceversa.

\section{Modelación desde la Socioepistemología}

La TSE propone una trasformación del paradigma imperante en la educación matemática. Este se basa en el cambio de centración de los conceptos matemáticos a las prácticas sociales y usos del conocimiento matemático.

Se han estudiado fenómenos que ocasiona el discurso matemático escolar (dME), como: la exclusión, la opacidad y la adherencia (Cordero, Gómez, Silva-Crocci \& Soto, 2015). Estos se producen por la hegemonía de las argumentaciones y significaciones de las nociones matemáticas, lo que no permite que los sujetos construyan el conocimiento matemático.

Cordero (2016) propone el programa "Sujeto olvidado y transversalidad de saberes" (SOLTSA). Este consiste en revelar los usos del conocimiento matemático y sus resignificaciones en las comunidades de conocimiento matemático de la gente: en la escuela, en el trabajo o la profesión, y en las vidas cotidianas (Mendoza-Higeras \& Cordero, 2018, p.42).

EI SOLTSA concibe a la modelación como una práctica social que ha permitido al humano; hombres y mujeres, construir conocimiento matemático. En este sentido, cuestiona a las aproximaciones que versan

Sistema de razón que norma las prácticas y representaciones sociales de los sujetos en torno a la enseñanza y el aprendizaje de las matemáticas (Soto y Cantoral, 2014). 
sobre la modelación considerando como referente, la acción o las estructuras cognitivas que se realizan en escenarios científicos para problematizar y sistematizar los elementos que intervienen cuando se modela.

Desde esta perspectiva la modelación permite la horizontalidad de las relaciones reciprocas entre el conocimiento matemático y el conocimiento de la gente. Por tanto, permite amortiguar los efectos del dME,

La propuesta para el diseño de situaciones de modelación en la FIDM se generó considerando cuatro constructos de la TSE; la problematización del saber matemático escolar, la comunidad de conocimiento, el uso de la gráfica y los diseños de situaciones de modelación.

\section{La problematización del saber matemático}

Reyes- Gasperini (2016) señala que "Cuando se lleva a cabo la problematización del saber matemático se tiene como objetivo entender los usos y razón de ser del conocimiento matemático estudiado" (p. 54). Señala que esta es una metodología que considera el estudio de las cuatro dimensiones articuladas que abarca la TSE; didáctica, cognitiva, epistemológica y social.

Esta autora, propone cuatro interrogantes para el desarrollo de la problematización del saber matemático, las cuales se expresan en la siguiente imagen, considerando el orden de las dimensiones.

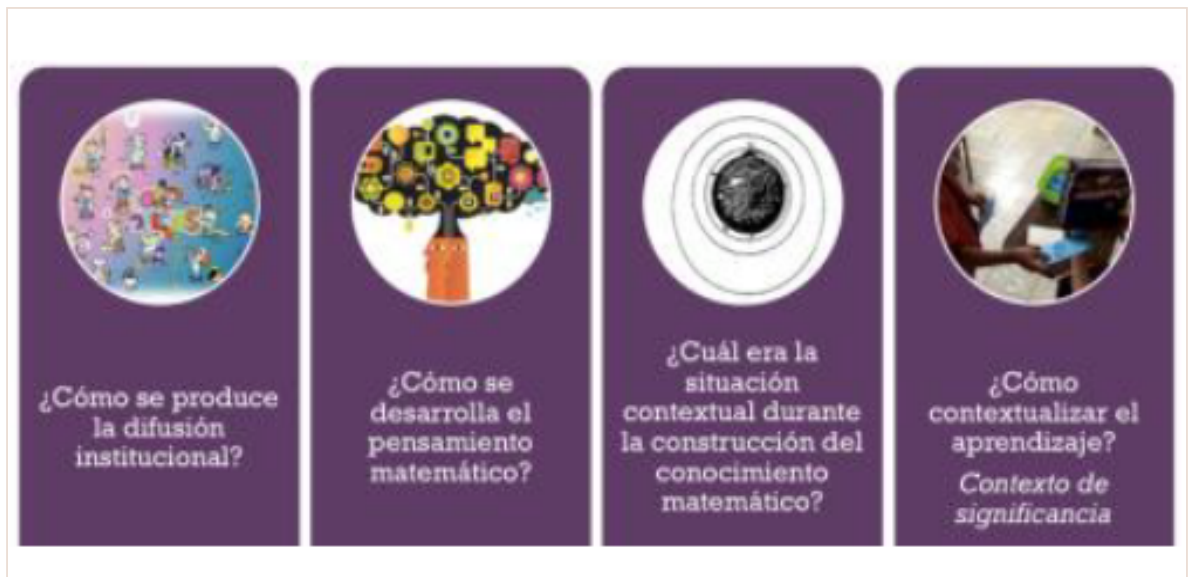

Ilustración 4. Cuestionamientos propuestos para la problematización del saber matemático (Reyes-Gasperini, 2016, p.56). 
Estas interrogantes permiten guiar la problematización del saber matemático. Para la propuesta, la problematización se desarrolla en términos de la modelación, por tanto los FPM discuten y reflexionan estas interrogantes a la luz de referentes teóricos que aborden la modelación en la ME.

\section{La comunidad de conocimiento}

Desde la TSE se han estudiado variedad de comunidades y escenarios; el histórico epistemológico, el cotidiano, los escenarios culturales, de la escuela y en dominios disciplinares diversos. Por ejemplo, se ha documentado la construcción de conocimiento de la comunidad sorda, Otomie, Ñuu savi, ingenieros, entre otras (Cordero, Méndez, Parra \& Pérez, 2014)

De estos estudios han emergido situaciones que expresan el Uso del Conocimiento Matemático (UCM); situación de variación, de aproximación, de transformación y de selección (Cordero, Del Valle \& Morales, 2019).

Méndez, Parra, Opazo, Pérez \& Cordero (2015) señalan que los UCM son construidos en comunidad a partir de consensos, de su cultura y de una situación específica. Aseveran "si hay conocimiento hay una comunidad que lo construye". La comunidad de conocimiento matemático se caracteriza a partir de la triada: localidad, intimidad y reciprocidad, estos en relación al UCM. Además se considera el papel de la institucionalización para la permanencia del UCM, así como la identidad de la comunidad, entendiéndola como la fuente de sentido para la construcción de conocimiento (Cordero \& SilvaCrocci, 2012).

A modo de visualización se muestra el modelo de comunidad de conocimiento 


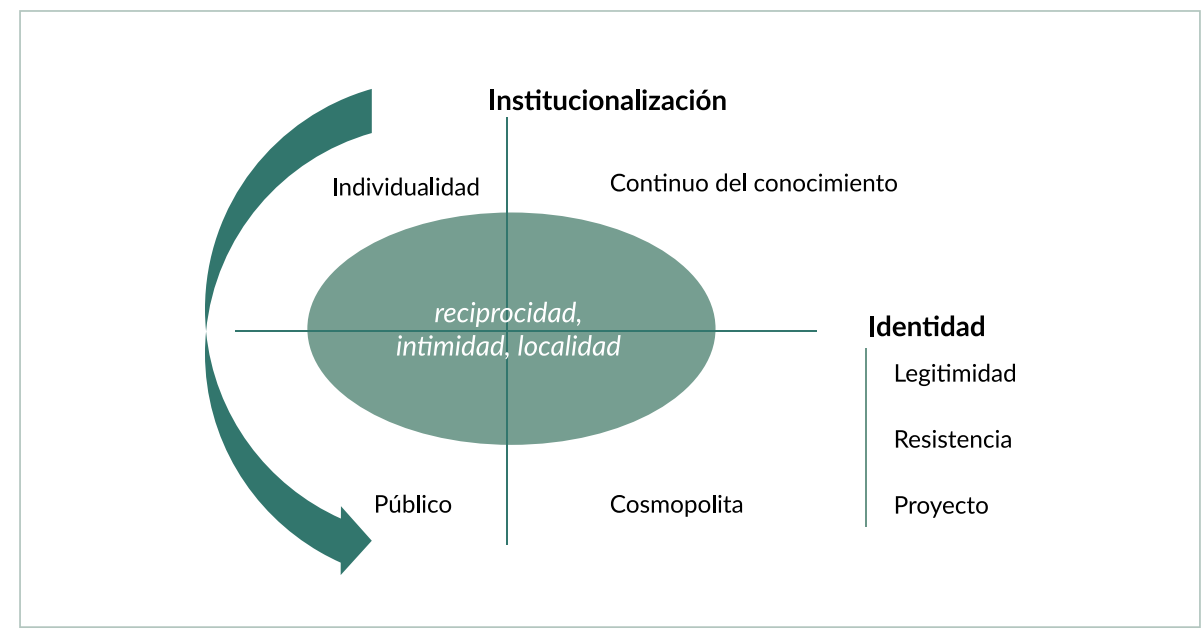

Ilustración 5. Comunidad de conocimiento (Cordero, 2016).

Localidad: Se entiende que el conocimiento es local y se genera cuando hay coincidencia de ideas, una jerga disciplinar o una concepción común. Esto contrasta con la idea de cosmopolita (conocimiento compartido por todo el mundo).

Intimidad: Se refiere a los argumentos íntimos de la comunidad con respecto a la construcción de conocimiento. Estos son privados, se comparte solo con la comunidad que los genera.

Reciprocidad: Se concibe que la construcción de conocimiento no es individual, sino de un conjunto de personas que deben generar un compromiso mutuo con ese conocimiento.

Para la propuesta se promueve el reconocimiento de una comunidad, con el fin de que los FPM puedan identificar sus características propias y diseñen situaciones de modelación de acuerdo a cada realidad particular.

\section{El uso de la gráfica}

La TSE a promovido un nuevo estatus al uso de la gráfica, proponiéndola como una práctica social que permite la construcción del conocimiento matemático (Morales \& Cordero, 2014).

Cordero (1998) establece una categoría que le permite establecer relaciones entre funciones, el uso de la gráfica y la modelación, esta categoría 
se denomina comportamiento tendencial de las funciones. A la luz de esta, se han desarrollado diseños que promueven la identificación coeficientes de la función, el reconocimiento de patrones de comportamientos gráficos, la búsqueda de tendencias en los comportamientos y la relación entre funciones, y comportamientos de fenómenos específicos.

Las investigaciones sobre el uso de la gráfica en el dME, dan cuenta de que ha quedado en un estatus de representación, ya sea de las funciones o para el ordenamiento de datos (utilización típica en estadísticas), esto ha soslayado el hecho de que en la epistemología del conocimiento y en el cotidiano, el uso de la gráfica ha permitido la construcción de nuevo conocimiento (Cordero, Cen \& Suarez, 2010; Cordero \& Flores, 2007). Si se observa el currículo actual de Matemáticas o los textos de estudio, la gráfica solo aparece como un apoyo a las nociones estudiadas. En tanto, que permite la visualización y es una representación que conceptualiza conceptos matemáticos. La argumentación gráfica no es válida dentro del dME, la argumentación algebraica ha imperado en la escuela.

El estudio desarrollado por Cordero \& Flores (2007) muestra que si bien la gráfica es explicita a partir de la enseñanza de las funciones o en estadística, el uso de la gráfica aparece desde los primeros niveles de enseñanza, se reconoce un síntoma de la gráfica. Es decir, su uso es transversal en la enseñanza de la Matemática. La gráfica transita desde los niveles básicos hasta llegar a la enseñanza de nivel superior, por ejemplo, en la resolución de ecuaciones diferenciales.

Se ha señalado que la construcción del cálculo se apoyó, en un primer momento, a partir de argumentaciones gráficas (Suarez \& Cordero, 2010). Donde la modelación de fenómenos de la naturaleza, específicamente el estudio del cambio, permitió la emergencia de nociones matemáticas como la derivada, la integral, entre otras.

Suárez \& Cordero (2010) reportan que la graficación es argumentativa desde el estudio de la obra de Oresme; este usaba figuras geométricas para describir una cualidad del movimiento. La manera en que Oresme justificó esta cualidad es tomando un punto de una línea horizontal, y levantar una perpendicular a esta línea para representar el cambio de intensidad, es decir, el cambio de cantidad de movimiento (Briceño, 2013).

Para el diseño de la situación de modelación se promueve el uso de la gráfica y el comportamiento tendencial de las funciones, ya que permite 
a los estudiantes generen argumentos para el diseño de la situación de modelación, reconociendo transversalidad y la argumentación gráfica.

\section{Diseños de situaciones de modelación}

Un referente que se ha considerado desde la TSE para la construcción de situaciones es Berger \& Luckmann (2006), quienes caracterizan la construcción social de la realidad. Estos autores señalan la importancia de reconocer el mantenimiento de rutinas y de crisis. Zaldivar (2014) reinterpreta estas nociones y las considera como el mecanismo que podría dar cuenta del cotidiano de la gente en la construcción del conocimiento matemático.

Briceño (2013) y Zaldívar (2014) han diseñado situaciones de modelación con tecnología, a partir de los usos de la gráfica en situación específica del movimiento. A partir de momentos de rutinas y crisis los sujetos de estudio realizan acciones, organizan comportamientos, proponen hipótesis y resignifican sus conocimientos matemáticos. Un resultado de estas investigaciones, ha sido el tránsito de la noción de movimiento desde una concepción de trayectoria, que expresa el cotidiano de la gente, al estudio de los fenómenos en un eje de coordenadas a partir de la distancia y el tiempo.

Méndez (2016) señala que los diseños de situación de modelación escolar deben considerar:

a) La experimentación; de donde se obtienen datos y tienen sentido el estudio de las condiciones iniciales y el comportamiento global del fenómeno. Esto da significado a los dominios y recorrido de las funciones.

b) El estudio de las variaciones locales y globales de gráficas o tablas numéricas.

c) La descripción, análisis y ajuste de comportamientos que transforman los datos en los modelos generados. Esto permite predecir el fenómeno. 


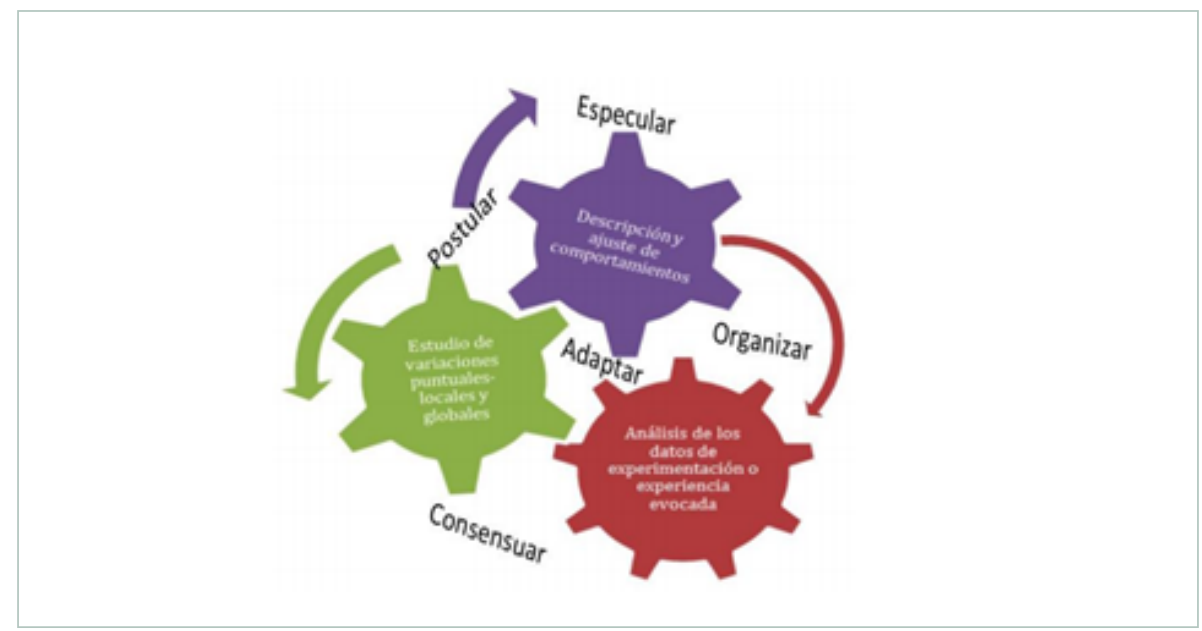

Ilustración 6. Méndez, Marquina, Zúñiga (2017).

En síntesis, las situaciones de modelación que será diseñadas por los FPM consideraran el mantenimiento de rutinas y crisis, experimentaciones, análisis global y local de las gráficas y transformaciones a los datos que permitan ajustar el modelo al fenómeno o el fenómeno al modelo.

\section{Propuesta para el diseño de situaciones de modelación en la FIDM}

El curso de didáctica del algebra y del cálculo (DAC) se dicta en el quinto semestre del plan de estudio de la carrera de Pedagogía en Matemática y Computación de la Universidad de Santiago de Chile.

La asignatura contempla cinco unidades que responde a los siguientes resultados de aprendizaje:

- Identifica a la Didáctica de la Matemática como dominio científico.

- Problematiza la modelación en la educación matemática las dimensiones, cognitiva, didáctica, social y epistemológica.

- Diagnostica una comunidad educativa e interpreta la información acerca de las necesidades en torno a la modelación.

- Diseña una situación de modelación a partir del diagnóstico de la comunidad y la problematización del saber. 
- Aplicar la situación de modelación y analizar los resultados en conjunto con la comunidad educativo.

De acuerdo a estos resultados de aprendizaje se organiza la propuesta para el diseño de situaciones de modelación de los FPM, en tres fases; problematización de la modelación, reconocimiento de la comunidad y diseño, aplicación y análisis de la situación de modelación. Estas fases están articuladas; cada una de ellas robustece y permite reflexionar sobre las otras.

Se presenta un esquema de la propuesta con sus respectivos objetivos:

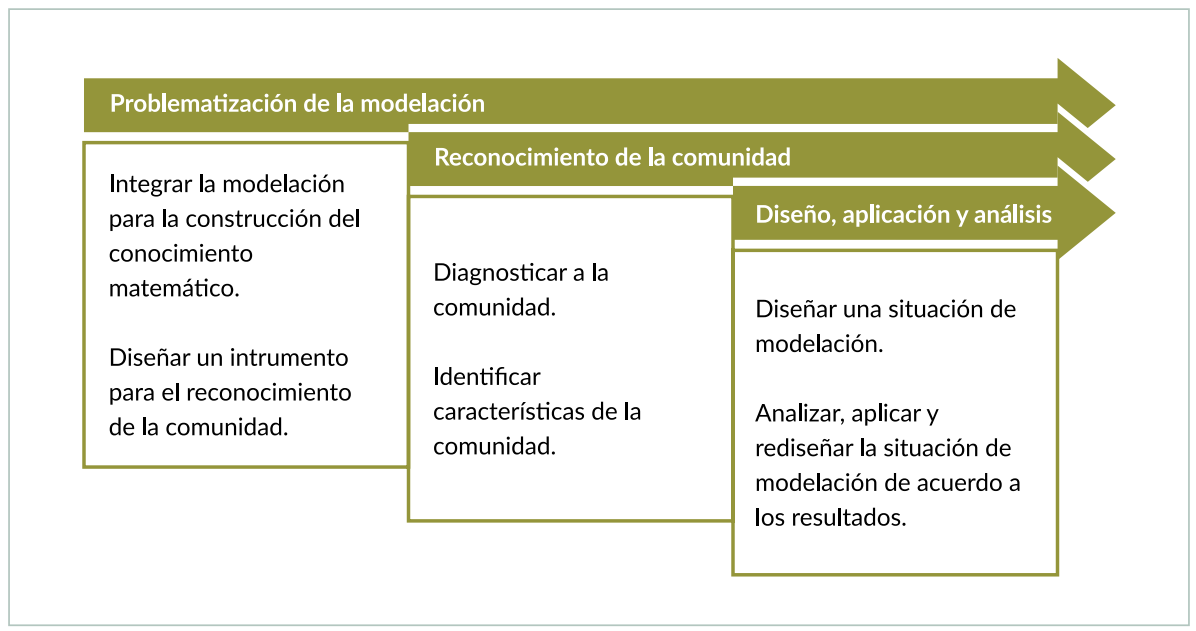

Ilustración 7. Propuesta para el diseño de situaciones de modelación en la FIDM.

\section{- Problematización de la modelación}

Esta fase tiene por objetivo que los FPM integre la modelación para la construcción del conocimiento matemático y diseñen un instrumento que permita el reconocimiento de una comunidad específica, a este se le denomina "diagnóstico".

Se problematizan la modelación desde las cuatro dimensiones del saber matemático. Las tareas en esta fase consisten en discutir, reflexionar y contrastar diferentes investigaciones de la modelación. Se reflexiona sobre el uso de la gráfica, el rol el docente frente a una situación de modelación, la relación matemática y realidad, el bipolo modélico, ciclos de modelación, entre otras perspectivas. 
También se problematiza el saber matemático escolar, en particular se discuten sobre nociones del algebra y del cálculo a la luz de la modelación, como: derivada, función cuadrática, función lineal, ecuaciones, sistemas, entre otras.

\section{- Reconocimiento de la comunidad}

Esta fase tiene como objetivo caracterizar la comunidad e identificar características de la comunidad que permitan argumentar el diseño de la situación de modelación.

Esta caracterización se desarrolla a partir de los elementos del modelo de comunidad de conocimiento; la localidad, intimidad y reciprocidad. Al ser una primera aproximación a la comunidad, los FPM plantean el diagnóstico en torno a los usos de la modelación en la clases de matemáticas y en su cotidiano. De esta forma, la localidad se expresa en la coincidencia de concepciones sobre las habilidades necesarias para el aprendizaje de las matemáticas. La intimidad, se expresa en los argumentos de los estudiantes con respecto a los usos de la modelación en la clase de matemáticas y en su cotidiano. Y, por último, la reciprocidad se observa en el compromiso mutuo de los estudiantes ante la resolución de problemas de su entorno.

Para esta etapa es necesario establecer un convenio con un establecimiento educacional, donde los agentes de la comunidad educativa (directivos, profesores y estudiantes) estén involucrados en el proyecto. Se debe establecer a priori una relación recíproca, que permita a los FPM reconocer a la comunidad y la aplicación del diagnóstico y sus diseños. Y a la comunidad sentirse parte de la reflexión. Para esto los profesores de matemática de la comunidad revisan los diseños y al finalizar el proyecto se les entrega el conjunto de diseños, que fueron perfeccionados a partir del análisis de su aplicación.

\section{- Diseño, aplicación y análisis}

De acuerdo a lo problematizado y los diagnósticos desarrollados, se proponen diseños de situación de modelación. La utilización de la tecnología es un aspecto que norma el diseño, al igual que la ingeniería didáctica para el análisis de su aplicación. 


\section{Resultados}

La aplicación de la propuesta se desarrolló primer semestre 2018. El curso estuvo compuesto por 18 FPM, los cuales conforman equipos para efectuar el trabajo de la asignatura. Se considera el proceso descrito por el equipo conformado por tres futuras profesoras de matemáticas, quienes diseñaron la situación: ¿Por qué ponemos sal al hielo? Se tomarán extractos de lo que ellas escriben en su informe final, los que serán destacados en formato de cita textual, sin referenciar al documento.

Durante la asignatura los estudiantes reciben retroalimentación constante de parte de la docente a cargo de la asignatura, se contemplan presentaciones orales del diagnóstico y del diseño de situación de modelación, reflexiones escritas a partir foros de discusión en la plataforma Moodle. Y la entrega de dos informes; un informe inicial, en este se problematiza la modelación y se propone el diagnóstico. Y el informe final, que es una extensión del primer informe. En este se robustece la problematización, de acuerdo a los resultados del diagnóstico, se presentan la situación, los resultados de su aplicación y el rediseño.

\section{Problematización de la modelación}

Al problematizar, las FPM integran a la modelación como una práctica que les permite promover la construcción de conocimiento matemático, comparar modelos para proponer diseños de modelación en el aula e identificar las problemáticas documentadas para el desarrollo de la modelación en la clase de matemáticas.

Para esto se consideran variados referentes teóricos, algunos discutidos en la asignatura y otros resultados de la búsqueda autónoma, entre ellos: Villa-Ochoa (2014) sobre el rol del profesor de matemática en el proceso de modelación, Arrieta y Díaz (2016) reconociendo las nociones de lo modelado y el modelo. Además de vincular las ideas provenientes de los referentes, con lo dispuesto por el currículo nacional.

Al sintetizar la problematización de la modelación desde las cuatro dimensiones, las FPM presentan una tabla que permite la explicitación de las preguntas y tareas para el diagnóstico: 
Tabla 1

Problematización de la modelación desde las cuatro dimensiones.

\begin{tabular}{|c|c|c|c|c|}
\hline DIMENSIONES & LO COGNITIVO & LO DIDÁCTICO & LO EPISTEMOLÓGICO & LO SOCIAL \\
\hline & $\begin{array}{l}\text { ¿Qué } \\
\text { habilidades } \\
\text { crees que son } \\
\text { necesarias } \\
\text { para aprender } \\
\text { matemática? } \\
\text { ¿Qué } \\
\text { estrategiaso } \\
\text { procedimientos } \\
\text { tienes para } \\
\text { lograr resolver } \\
\text { problemas } \\
\text { matemáticos? }\end{array}$ & $\begin{array}{l}\text { ¿Has visto en } \\
\text { el texto del } \\
\text { estudiante } \\
\text { situaciones de } \\
\text { modelación? } \\
\text { En las clases de } \\
\text { matemática } \\
\text { ¿Has discutido } \\
\text { sobre la } \\
\text { modelación? }\end{array}$ & $\begin{array}{l}\text { Reconocimiento de } \\
\text { patrones: } \\
\text { El estudiante } \\
\text { debe identificar el } \\
\text { cambio, la variación } \\
\text { y luego, predecir. } \\
\text { ¿Cómo crees } \\
\text { que se utilizan } \\
\text { las funciones } \\
\text { cuadráticas } \\
\text { en el estudio } \\
\text { de fenómenos } \\
\text { Naturales? }\end{array}$ & $\begin{array}{l}\text { ¿Reconoces } \\
\text { algún objeto } \\
\text { matemático en } \\
\text { las actividades } \\
\text { que realizas } \\
\text { en tu vida } \\
\text { cotidiana? } \\
\text { ¿Crees que la } \\
\text { matemática } \\
\text { te ayuda a } \\
\text { encontrar } \\
\text { una solución } \\
\text { a algunos } \\
\text { problemas de tu } \\
\text { vida cotidiana? }\end{array}$ \\
\hline
\end{tabular}

La problematización les permitió a las FPM, confeccionar el diagnóstico con el objetivo de indagar sobre el uso de la modelación en la clase de matemáticas.

El diagnóstico realizado a los alumnos es construido con el objetivo de obtener respuestas con las cuales nos permitirán evaluar la carencia de la modelación tanto en el aula de clases, como en el vocabulario de los alumnos y ya con estos datos poder realizar la construcción de nuestro diseño de situación.

También se propusieron caracterizar las habilidades de los estudiantes con respecto al reconocimiento de patrones o regularidades, provenientes de una realidad especifica.

El objetivo de construir estos modelos es que los estudiantes descubran ciertas regularidades y/o patrones, además de que puedan expresar esas características, desarrollando la capacidad de razonary resolver problemas. 


\section{Reconocimiento de la comunidad}

Los resultados del diagnóstico permitieron a las FPM reconocer algunas características cualitativas la comunidad de conocimiento estudiada.

La comunidad de conocimiento en la cual se desarrolla el estudio está ubicada en Santiago de Chile, es un establecimiento educacional científicohumanista y se caracteriza por ser una institución de varones. Las actividades se desarrollaron con un curso de $2^{\circ}$ año medio (15-16 años), se organizó cada equipo de FPM con un grupo de 5 estudiantes, a estos se les aplicó el diagnóstico y luego la situación de modelación.

En cuanto a la localidad, los resultados del diagnóstico han provisto de las concepciones de la comunidad sobre las habilidades para el aprendizaje de las matemáticas.

A pesar de que el currículo escolar fomenta el trabajo de las habilidades en las actividades propuestas en los textos de matemáticas, los alumnos al preguntarles por las habilidades que son necesarias para aprender matemáticas, automáticamente nos dicen que la memorización es una de ellas, reflejando que la metodología de enseñanza es estructurada y conductista, la cual fomenta sólo la memorización, lo que trae como consecuencia la ausencia de situaciones en donde coloquen en práctica, por ejemplo, la modelación matemática, permitiendo que interioricen el significado del objeto matemático tratado comprendiendo la funcionalidad de cada uno de ellos y utilizarlos en lo cotidiano según el contexto en el que se enfrente cada uno de ellos.

La intimidad de la comunidad se ve expresada en el uso de la modelación en la clase de matemáticas y en su cotidiano.

[..] Pudimos identificar que la modelación no es algo habitual para ellos, aun así, reconocen implementarlo en el día a día en su cotidiano, es por esto por lo que buscamos presentarles la modelación de una manera diferente.

La reciprocidad de la comunidad, se expresó el compromiso mutuo de los estudiantes ante tareas específicas de reconocimiento de patrones y regularidades. Para esto las FPM se apoyan del programa Plickers, lo que les permitió motivar a los estudiantes a resolver y captar sus respuestas. 


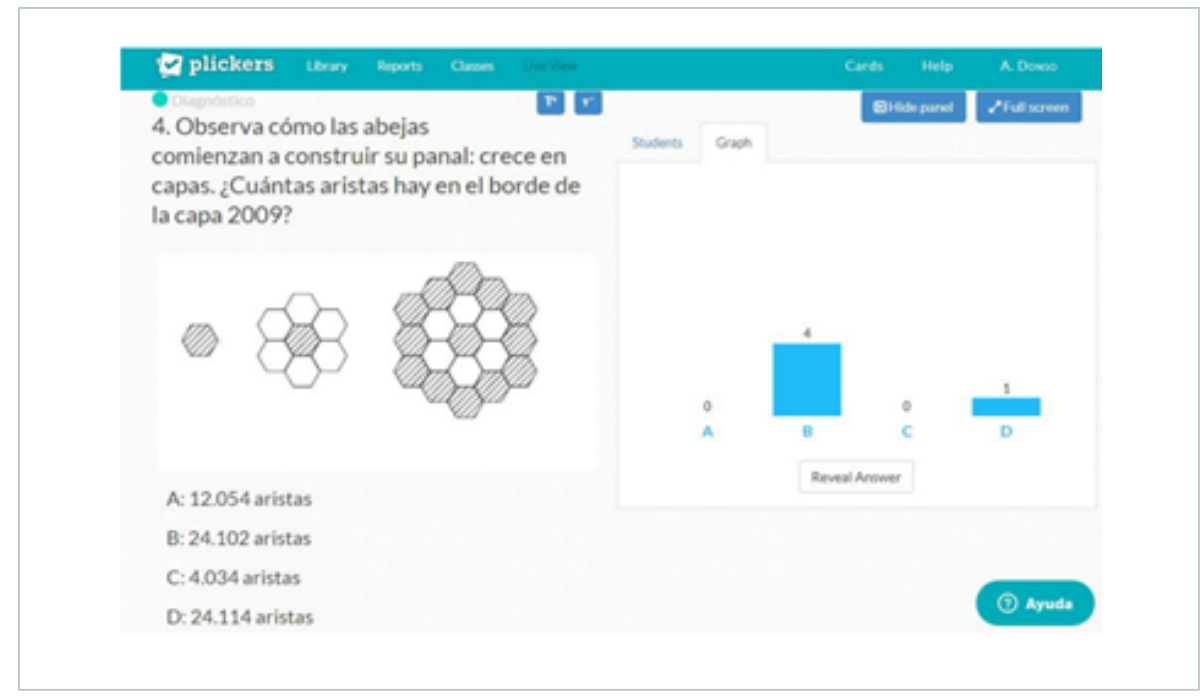

Ilustración 8. Imagen de una pregunta del diagnóstico.

El resultado del diagnóstico de la comunidad permite a las FPM identificar las características de la enseñanza de las matemáticas en esta comunidad, la carencia de la modelación en sus prácticas de enseñanza y el compromiso de los estudiantes por resolver tareas de reconocimiento de patrones y regularidades. De esta forma, las FPM plantean con su diseño de situación de modelación, provocar un quiebre en las visiones tradicionales de los estudiantes sobre la relación entre la matemática, la modelación y la vida cotidiana. Y fortalecer el compromiso por la resolución de problemas.

\section{Diseño, aplicación y análisis}

La situación de modelación que propuso el equipo de FPM, propone estudiar el fenómeno químico que se produce cuando se agrega sal a un vaso con agua y hielo. Las FPM pretenden provocar que los estudiantes desarrollen análisis locales y globales de la gráfica generadas al experimentar con un sensor de temperatura en vasos con agua, hielo y sal, de tal manera de generar un análisis de la variación de la temperatura en el tiempo.

Para esto consideran nociones provenientes de la TSE: mantenimiento de rutinas y crisis, y el uso de la gráfica y el comportamiento tendencial de las funciones. 
Para profundizar la situación, se consideró la noción del comportamiento tendencial de las funciones, ya no estaremos únicamente en el contexto algebraico, ahora nos sumergimos en los elementos didácticos, donde se pondrá en juego, lo algebraico con lo gráfico.

Señalan que el uso de la gráfica, les permite resignificar las nociones matemáticas involucradas en el diseño.

El usar las gráficas nos permite dar nuevos significados a una noción matemática en donde permite establecer una conexión entre la modelación y graficación, esto quiere decir que no sólo va a graficar para cumplir con lo didáctico, sino que podrá resignificar está continuamente y de esta manera generar su conocimiento matemático.

Las FPM señalan que la actividad comienza con dos preguntas: ¿Por qué se vierte sal en las carreteras para evitar que se hielen?, ¿Por qué si queremos enfriar rápidamente unas bebidas, ponemos sal en el hielo?

Para responder a esas preguntas, los alumnos y alumnas participan en una actividad de modelación en la cual trabajan (desde lo ) a nivel matemático y químico, colocando en práctica además las cuatro habilidades planteadas por el currículum, pero especificamente la habilidad de modelar, pues mediante una situación cotidiana aplicada a un contexto matemático, les permitirá llegar a conclusiones[..] hacia un elemento muy particular: el agua.

El diseño propuesto por las FPM está organizado en tres momentos: Contextualización, análisis y explicación. En el momento de contextualización se plantea ¿por qué cuando una carretera está helada hay camiones esparciendo sal? Se muestra a través de vídeos e imágenes de Internet la situación planteada. Se espera que el alumnado exprese la idea inicial de que la sal derrite el hielo.

Luego se propone las siguientes preguntas:

Si queremos enfriar unas bebidas con hielo, ¿añadimos sal al hielo para enfriar más rápidamente la bebida? ¿Qué le pasa al hielo cuando se le añade sal? 
En el informe las FPM señalan que en el primer momento, los estudiantes deben interactuar con los distintos recipientes. Se tendrán cinco recipientes en total, de los cuales uno tendrá agua, dos con agua y hielo, y dos con hielo. Se hace énfasis en el dialogo de los estudiantes en torno a la pregunta y predicción sobre el comportamiento de cada vaso antes de agregar el factor de cambio; la sal.

Las FPM plantean que en este momentos lo importante son las hipótesis de los estudiantes, las cuales, deberían ir cambiando a medida que se realice una crisis entre cada momento.

En el momento de experimentación y análisis, las FPM señalan que el grupo de estudiantes usará los vasos y la calculadora con el sensor de temperatura para registrar los cambios de temperatura que presenta cada recipiente sin incorporar la sal. Contrastando lo que predijeron al inicio de la actividad con el comportamiento visto mediante la calculadora.

Se desarrollará una crisis cuando se incorpore sal a uno de los recipientes que posee hielo con agua y otro que posee solo hielo.

Las FPM señalan que posteriormente los estudiantes deben registran los datos en una tabla, esto les permitirá comparar las temperaturas observadas. Además, se mide la temperatura ambiental para iniciar una discusión posterior, en la fase de reflexión, sobre la influencia de este factor. Las consignas del diseño son las siguientes:

Incorporar sal en uno de los recipientes que contiene: agua con hielo y solo hielo.

Utilizar la calculadora con los sensores de temperatura para registrar los cambios de temperatura con el transcurso de los minutos.

Mediante la calculadora (o de manera manual), registrar los datos en la tabla entregada.

\begin{tabular}{|c|c|c|c|c|c|}
\hline Tiempo & Agua & Agua hielo & $\begin{array}{c}\text { Agua hielo } \\
\text { y sal }\end{array}$ & Hielo & $\begin{array}{c}\text { Hielo con } \\
\text { sal }\end{array}$ \\
\hline & & & & & \\
\hline
\end{tabular}


En el momento de explicación, las FPM señalan que se les hará una serie de preguntas, esto tiene como objetivo resignificar la gráfica de una función, esta parte responde directamente a los cuestionamientos iniciales, ya que se preguntan por lo que sucede con los datos si se agrega mayor cantidad de sal.

1. Grafique el comportamiento del recipiente asignado.

2. Analice y compare los gráficos del grupo.

3. Responda.

- ¿Cómo será la gráfica del vaso con agua? ¿Qué nombre recibe ese tipo de función?

- ¿Qué recipiente presenta una mayor variación de temperatura con el transcurso del tiempo?

Por último, al final de la situación las FPM plantean un cuestionario abierto de reflexión final sobre el fenómeno:

- ¿El hielo se derrite o se disuelve con la sal?

- ¿Ustedes creen que pasa lo mismo con el azúcar?

- ¿Qué situación elegirían para disolver el hielo?

- ¿La temperatura afecta en la disolución del hielo?

- ¿Qué pasa con el volumen del agua?

- ¿Por qué el río se congela si es de agua dulce?

\section{Experimentación y análisis}

Para el desarrollo del análisis, la aplicación y el análisis se consideró la ID, de esta forma las FPM reportaron sobre el análisis a priori, a posteriori y la confrontación.

Las FPM señalan las respuestas esperadas y lo confrontan con lo que sucedió en la aplicación.

La actividad comenzó con las siguientes preguntas: ¿a qué temperatura se congela el agua? ¿A qué temperatura hierve el agua?, la cual permitió al alumno orientarse en el contexto en 
el cual se trabajó a lo largo de la actividad, se esperaba que los alumnos nos expresaran inicialmente que el agua se congela a o grados, y hierve a 100 grados, esto, rápidamente fue contestado por ellos de la manera esperada.

Describen el momento de crisis, y la confirmación de sus hipótesis hechas en el análisis a priori:

Se creará una crisis en donde le incorporaremos un factor cambio al hielo, el cual sería la sal, por lo que nos permite realizar la siguiente interrogante, ¿qué sucede si le incorporamos sal al hielo?

En el transcurso de la actividad, podremos confirmar o refutar nuestras hipótesis de respuestas por parte de los alumnos, pues al observar las gráficas y las características que poseía cada recipiente antes de incorporar la sal, y realizar una comparación.

En la ilustración 9 se muestran algunas de las gráficas construidas por los estudiantes durante la aplicación de la situación.

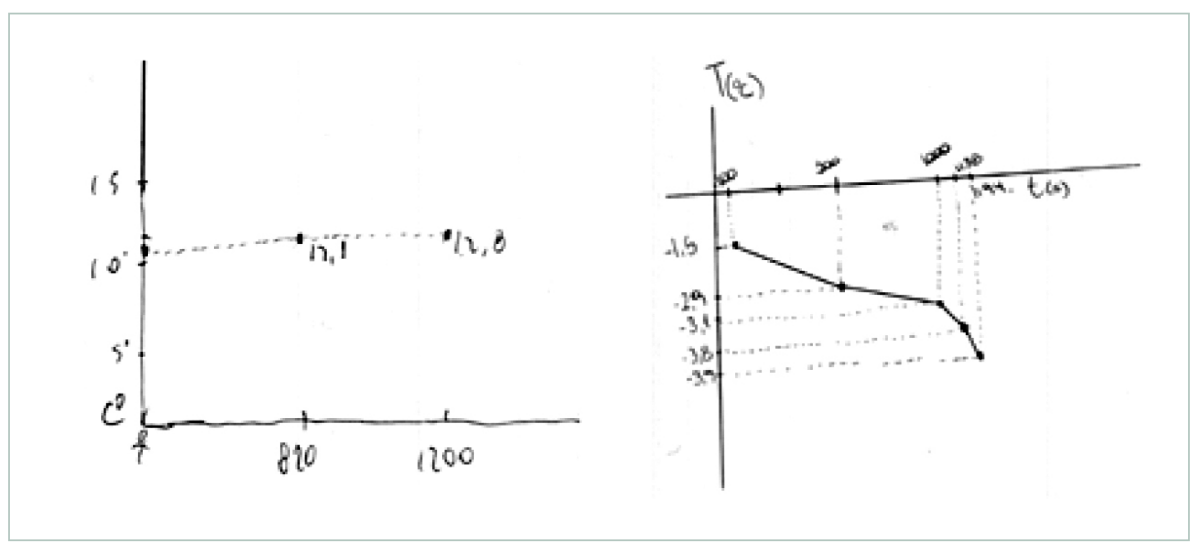

Ilustración 9. Gráfica de agua a temperatura ambiente y agua con hielo y sal.

Terminan el informe, valorando la situación de modelación y la disciplina de la ME para la construcción de diseños de situaciones de modelación

Se puede mencionar que, mediante las investigaciones realizadas y la experimentación de la situación, encontramos una actividad la cual nos permite resignificar las funciones. [..] Es importante saber que existe esta manera de enseñar matemática, ya que podríamos 
decir que la gran mayoría de las problemáticas detectadas por la ME son efectos de este punto [..]

Además anexan algunas fotografías de la experiencia.
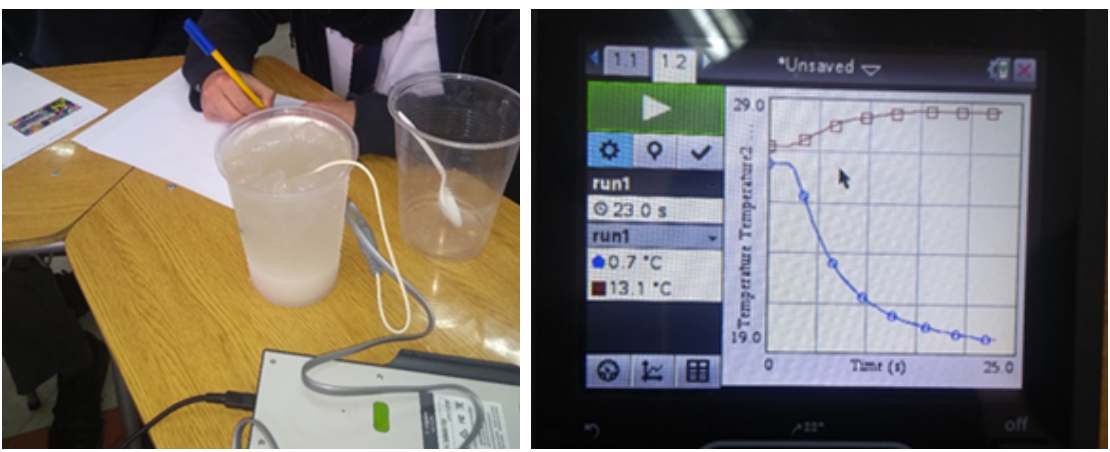

Ilustración 70. Fotografías de la aplicación del diseño de situación de modelación.

\section{Reflexiones finales}

El objetivo de este documento fue presentar una propuesta para el diseño de situaciones de modelación en la FIDM. La intención es que esta propuesta se pueda replicar en asignaturas de didáctica que busquen el desarrollo de diseños de modelación por parte de los FPM, desde el reconocimiento de comunidades específicas.

La propuesta considera tres fases interconectadas, estas son: problematización de la modelación, reconocimiento de la comunidad, y diseño, aplicación y análisis de una situación de modelación.

La problematización de la modelación permite que los FPM logren integrar a la modelación como un proceso para la construcción del conocimiento matemático y diseñar un instrumento diagnóstico para el reconocimiento de una comunidad especifica. En este proceso las FPM se hacen sensibles hacia la problemática de la inclusión de la modelación en el aula. A través de su experiencia como FPM y la reflexión sobre las lecturas del curso, formulan hipótesis acerca de la comunidad que diagnosticaran. 
Las FPM señalan la necesidad de reconocer la carencia de la modelación en el aula de matemática de la comunidad estudiada, las habilidades de los estudiantes para el reconocimiento de patrones y regularidades, y las formas tradicionales de la enseñza aprendizaje de las matemáticas en esta comunidad.

El reconocimiento de la comunidad tiene como propósito que las y los FPM diagnostiquen una comunidad e identifiquen sus características, para argumentar la situación de modelación que más tarde diseñaran. En esta fase las y los FPM deben distinguir, organizar información, explicar las causas de las respuestas de los estudiantes y sintetizar la información extraída del diagnóstico para el diseño de una situación de modelación. Los resultados del diagnóstico permiten reconocer en la comunidad; La concepción de que la memorización es una habilidad para el aprendizaje de las matemáticas, el escaso uso de la modelación en la clase de matemática, el reconocimiento de la matemática en el cotidiano y el compromiso por resolver problemas de su entorno, a partir del reconocimiento de regularidades y patrones. Esto denota la localidad, la intimidad y la reciprocidad en esta comunidad de conocimiento.

La síntesis del diagnóstico fue: generar una situación de modelación que provoque en los estudiantes desarrollar experimentación ante una problemática cotidiana. Esto permite quebrar la idea de que la memorización es la habilidad para el aprendizaje de las matemáticas. El diseño promueve la argumentación gráfica para la construcción de conocimiento matemático.

Si bien la fase de reconocimiento de la comunidad, permitió a las estudiantes identificar el uso de la modelación en la asignatura de matemática de esta comunidad y algunas concepciones sobre la enseñanza de las matemáticas, se considera, para futuras aplicación, de la propuesta, profundizar en las prácticas de la comunidad que permitan reconocer contextos de interés, para el diseño de las situaciones de modelación.

En el diseño, aplicación y análisis se espera que los FPM diseñen una situación de modelación, la apliquen con los mismos estudiantes que diagnosticaron y la analicen a través de una ingeniería didáctica, lo que les permitirá cerrar con un rediseño de su situación de modelación.

Para el diseño se hace necesario reconocer un fenómeno que permita ser estudiado con la tecnología que se cuenta. Además, se deben generar momentos de rutinas y crisis que permita a los estudiantes generar hipótesis 
y conjeturas. Validando o refutando a partir de la toma de datos. Esta fase promueve la creatividad de las y los FPM.

De esta forma, se puede concluir que la propuesta de diseño de situaciones de modelación para la FIDM, ha permitido a los y las FPM generar diseños a través de una problematización de la modelación y el reconocimiento de una comunidad. Así mismo, obtener como resultado un conjunto de situaciones que fueron socializadas con los profesores de la comunidad.

Como prospectiva se destaca el estudio de la reproducibilidad de las situaciones de modelación en el aula, así como los impactos que puedan generar en la comunidad escolar.

\section{REFERENCIAS BIBLIOGRÁFICAS}

Alsina, À. (2010). El aprendizaje reflexivo en la formación inicial del profesorado: un modelo para aprender a enseñar matemáticas. Educación Matemática, 22(1), 149-166. Recuperado de http://www.scielo.org.mx/ pdf/ed/v22nt/v22n1a7.pdf

Aravena, M. (2016). Modelación matemática en Chile. En Arrieta, J., \& Díaz, L. (Eds.), Investigaciones latinoamericanas de modelación de la matemática educativa (pp. 195-233). Barcelona, España: Gedisa

Aravena, M., Caamaño, C., \& Giménez, J. (2008). Modelos matemáticos a través de proyectos. Revista Latinoamericana de Investigación en Matemática Educativa, 77(7), 49-92. Recuperado de https://www.redalyc. org/pdf/335/33511103.pdf

Berger, P., \& Luckmann, T. (2006). La construcción social de la realidad. Buenos Aires: Amorrortu.

Blomhøj, M. (2009). Different perspectives in research on the teaching and learning mathematical modelling-Categorising the TSG21 papers. In Blomhøj, M., \& Carreira, S. (Eds.), Mathematical applications and modelling in the teaching and learning of mathematics: Proceedings from TSG21 at the ICME17 (pp. 1-17). Dinamarca: Roskilde University. 
Blum, W., \& Borromeo-Ferri, R. (2009). Mathematical Modelling: Can It Be Taught And Learnt? Journal of Mathematical Modelling and Application, 7(1), 45-58. Recuperado de https://pdfs.semanticscholar.org/eb c2/4e810efa2f5361b9accfc0097c2bca084b89.pdf

Borromeo- Ferri, R. (2014). Mathematical Modeling -The Teacher's Responsibility. En B. Dick -Man, \& A. Sanfratello (Eds.), Proceddings Conference on mathematical modeling (pp. 26-31). New York, Estados Unidos: Columbia University.

Briceño, E. (2013). El uso de la gráfica como instrumento de argumentación situacional con recursos tecnológicos (Tesis doctoral). Cinvestav-IPN, D.F, México.

Cantoral, R. (2013). Teoría Socioepistemológica de la Matemática Educativa: estudios sobre construcción social del conocimiento. D. F., México: Gedisa.

Cordero, F. (2016). Modelación, funcionalidad y multidisciplinariedad: el eslabón de la matemática y el cotidiano. En Arrieta, J., \& Díaz, L. (Eds.), Investigaciones latinoamericanas de modelación de la matemática educativa (pp. 59-88). Barcelona, España: Gedisa

Cordero, F. (1998). El entendimiento de algunas categorías del conocimiento del cálculo y análisis: el caso del comportamiento tendencial de las funciones. Revista latinoamericana de Investigación en Matemática Educativa, 7(1), 56-74. Recuperado de https://www.redalyc.org/ pdf/335/33510105.pdf

Cordero, F., Gómez, K., Silva- Crocci, H., \& Soto, D. (2015). Discurso matemático escolar. Adherencia, exclusión y opacidad. Barcelona: Gedisa.

Cordero, F., Méndez, C., Parra, T., \& Pérez, R. (2014). Atención a la Diversidad: La Matemática Educativa y la Teoría Socioepistemológica. Revista Latinoamericana de Etnomatemática, 7(3), 71-90. Recuperado de https://www.redalyc.org/pdf/2740/274032530005.pdf

Cordero, F., \& Flores, R. (2007). El uso de la gráfica en el discurso matemático escolar. Un estudio sociepistemológico en el nivel básico a través de los libros de textos. Revista Latinoamericana de Investigación en Matemática Educativa, 10(1), 7-38. Recuperado de https://www.redalyc.org/pdf/335/33500102.pdf 
Cordero, F., \& Silva-Crocci, H. (2012). Matemática Educativa, Identidad y Latinoamérica. El quehacer y la usanza del conocimiento disciplinar. Revista latinoamericana de Matemática Educativa, 15(3), 295-318. Recuperado de http://www.scielo.org.mx/pdf/relime/v15n3/v15n3a3.pdf

Cordero, F., Del Valle, T., \& Morales, A. (2019). Usos de la optimización de ingenieros en formación: el rol de la Ingeniería mecatrónica y de la obra de Lagrange. Revista Latinoamericana de Investigación en Matemática Educativa, 22(2), 185-212. DOI: https://doi.org/10.12802/relime.19.2223

Cordero, F., Cen, C., \& Suárez, L. (2010) Los funcionamientos y formas de las gráficas en los libros de texto: una práctica institucional en el bachiIlerato. Revista Latinoamericana de Investigación en Matemática Educativa, 13(2), 187-214. Recuperado de http://www.scielo.org.mx/ pdf/relime/v13n2/v13n2a4.pdf

Giere, R. N. (1999). Using Models to represent reality. En L. Magnani, N.J. Nersessian, \& P. Thagard, Model-Based Reasoning in Scientific Discovery (pp. 41-57). Nueva York, Estados Unidos: Springer.

Huincahue, J., \& Mena-Lorca, J. (2014). Modelación matemática en la formación inicial de profesores. En C. Vásquez (Presidencia). Jornadas Nacionales de Educación Matemática: XIX JNEM 2015.

Huincahue, J., Borromeo, R., \& Mena-Lorca, J. (2018). El conocimiento de la modelación matemática desde la reflexión en la formación inicial de profesores de matemática. Enseñanza de las ciencias, 36(1), 99-115. Recuperado de https://www.raco.cat/index.php/Ensenanza/article/ view/335276

Huircán, M., \& Carmona, K. (2013). Guía de Aprendizaje No 3. Modelando el mundo con funciones exponenciales y logaritmos. Santiago de Chile: Ministerio de Educación. Recuperado de http://epja.mineduc.cl/ wp-content/uploads/sites/43/2016/04/201404141135550.GuiaN3MatematicallCiclodeEM.pdf

Kaiser, G., Sriraman, B., Blomhøj, M., \& Garcia, F. J. (2007). Report from the working group modelling and applications. Differentiating perspectives and delineating commonalties. En D. Pitta-Pantazi, \& G. Pilippou (Eds.), Proceedings of the Fifth Congress of the European Sociecty for Research in Mathematics Education (pp. 2035-2041). Chipre: University of Cyprus. 
Llinares, S. (2007). Formación de profesores de matemáticas. Desarrollando entornos de aprendizaje para relacionar la formación inicial y el desarrollo profesional. Conferencia invitada en la XIII Jornadas de Aprendizaje y Enseñanza de las Matemáticas - JAEM. Granada, Julio.

Llinares, S., Valls, J., \& Roig, A. I. (2008). Aprendizaje y diseño de entornos de aprendizaje basado en videos en los programas de formación de profesores de matemáticas. Educación matemática, 20(3), 59-82. Recuperado de https://www.redalyc.org/pdf/405/40512064004.pdf

Méndez, C., Opazo, C., Parra, T., Pérez, R., \& Cordero, F. (2015). Comunidad de conocimiento matemático. En R. Flores (Ed.), Acta latinoamericana de matemática Educativa, 28 (pp.1001-1108). México: Comité Latinoamericano de Matemática Educativa.

Méndez, M. (2016). Explorando la formación inicial. Reflexión sobre el diseño y aplicación de una situación de modelación escolar. En E. Mariscal (Ed.), Acta Latinoamericana de Matemática Educativa, 29 (pp. 11141121). México: Comité Latinoamericano de Matemática Educativa.

Méndez, M., Marquina, N., \& Zuñiga, K. (2017). Situaciones de aprendizaje para la modelación escolar. En L. Serna (Ed.), Acta Latinoamericana de Matemática Educativa, 30 (pp. 1046-1056). México: Comité Latinoamericano de Matemática Educativa.

Mendoza, E. J., \& Cordero, F. (2018). La modelación en las comunidades de conocimiento matemático. El uso de las matemáticas en ingenieros biónicos. El caso de la estabilidad. Revista Latinoamericana de Etnomatemática: Perspectivas Socioculturales de la Educación Matemática, 77(1), 36-61. Recuperado de https://www.researchgate.net/ publication/333738139_La_modelacion_en_las_comunidades_de_conocimeinto_matematico_El_uso_de_las_matematicas_en_ingenieros_bionicos_El_caso_de_la_estabilidad

MINEDUC. (2015). Bases curriculares $7^{\circ}$ básico a $2^{\circ}$ medio. Santiago: Ministerio de Educación de Chile.

MINEDUC. (2016). Matemática, Programa de estudio, primero medio. Santiago: Ministerio de Educación de Chile. 
Morales, A., \& Cordero, F. (2014). La graficación - modelación y la Serie de Taylor. Una socioepistemología del cálculo. Revista Latinoamericana de Investigación en Matemática Educativa, 17(3), 319-345. DOI: http:// dx.doi.org/10.12802/relime.13.1733.

Reyes-Gasperini, D. (2016). Empoderamiento docente desde una visión socioepistemológica: una alternativa para la transformación y la mejora educativa. (Tesis doctoral). Centro de Investigación y de Estudios Avanzados del IPN México.

Solar, H., Azcárate, C., \& Deulofeu, J. (2012). Competencia de argumentación en la interpretación de gráficas funcionales. Enseñanza de las Ciencias, 30(3), 133-154. Recuperado de https://ddd.uab.cat/pub/edlc/edlc_ a2012m11v30n3/edlc_a2012m11v30n3p133.pdf

Soto, D. Silva-Crocci, H. Barbe, J., \& Vergara, M. (2017). Prácticas educativas y el desarrollo de habilidades matemáticas: una propuesta de análisis para los instrumentos de evaluación de los docentes del Liceo Ruiz Tagle. En G. Watson, R. Fernández, \& G. Guerrero, Investigando juntos: experiencias asociativas entre escuelas y la Universidad de Santiago de Chile (pp. 70-78). Santiago: Universidad de Santiago de Chile.

Soto, D., \& Cantoral, R. (2014). El discurso Matemático Escolar y la Exclusión. Una visión Socioepistemologica. Bolema- Boletim de Educação matemática, 28(50), 1525-1544. DOI: https://doi.org/10.1590/19804415v28n50a25

Suárez, L., \& Cordero, F. (2010). Modelación - Graficación, una categoría para la matemática escolar. Resultados de un estudio socioepistemológico. Revista Latinoamericana de Investigación en Matemática Educativa, 13(4), 319 - 334. Recuperado de https://www.redalyc.org/ pdf/335/33558827006.pdf

Vargas, V., Escalante, C., \& Carmona, G. (2018). Competencias Matemáticas a través de la implementación de actividades provocadoras de modelos. Educación matemática, 30(1), 213-236. DOI: 10.24844/EM3001.08

Vilches, K. Soto, D., \& Silva-Crocci, H. (2019). Mathematical Modeling in Initial Teacher Training: An Epistemological Analysis. In F. Cordova, \& H. Rojas, Research in Education: Teacher Training Issues (pp. 55-84). Estados Unidos: Nova Science Publisher. 
Villa-Ochoa, J. A. (2014). Situaciones de modelación matemática, Algunas reflexiones para el aula de clase. Cuadernos de Investigación y Formación en Educación Matemática, 12, 281-290. Recuperado de https:// www.researchgate.net/publication/277140519_Situaciones_de_modelacion_matematica_Algunas_reflexiones_para_el_aula_de_clase

Villa-Ochoa, J., (2016). Aspectos de la modelación matemática en el aula de clase. El análisis de modelos como ejemplo. En J. Arrieta, \& L. Díaz, Investigaciones Latinoamericanas en Modelación Matemática Educativa (pp. 109-138). México: Gedisa.

Zaldivar. J. (2014). Un estudio de la resignificación del conocimiento matemático del ciudadano en un escenario no escolar (Tesis doctoral). Cinvestav-IPN, D.F, México. 